\title{
Sampled Data Control of a Compliant Actuated Joint Using On/Off Solenoid Valves
}

\author{
V. A. Tsachouridis ${ }^{*}, 1$, N. G. Tsagarakis ${ }^{2}$ and D. G. Caldwell ${ }^{2}$ \\ ${ }^{I}$ Author for correspondence; School of Technological Applications, Department of Industrial Informatics, Kavala Institute of Technology, \\ Agios Loucas, Kavala 65404, Greece. \\ ${ }^{2}$ Robotics, Brain and Cognitive Sciences Department, Italian Institute of Technology, Via Morego 30, 16163 Genoa, Italy.
}

Received 22 March 2009; Accepted 20 January 2011

\begin{abstract}
This paper proposes a new control system design method for a compliant actuated joint using on/off solenoid valves. The mathematical modelling and the system's hardware are described in detail. The control design method is presented in a general manner so it could be applied for any other similar system. For the present system, the designed controller is implemented via a digital computer and it is characterised by very good performance and simplicity. The success of the proposed method is validated via simulations and experiment.
\end{abstract}

Keywords: Actuators, Nonlinear Systems, Control Systems, Z-Transform, Optimization, Robots.

\section{Introduction}

In the 1950's, a new pneumatic system, known as the McKibben muscle was developed for applications in rehabilitation robotics [1]. At the time the power/weight performance of the system and the inherent compliance were seen as positive features but control was still a problem and the mechanism development was discontinued. In the 1980's Bridgestone in their Rubbertuator revived the principle of the McKibben muscle and this was incorporated into their "Soft arm" robot, which was produced for a number of years [2]. For these early versions of the actuator, models were developed and gave good accuracy over a broad range of forces and motions but errors of up to $20 \%$ in the force/displacement profile were not uncommon. By 1990's work on the Rubbertuator had stopped but several other groups had noted the potential of this form of actuation [3]-[5]. At the same time new applications were also being identified, particularly in the area of bio-robotics [6]-[8], but also with applications in unrelated domains such as the nuclear industry [9].

Over the 40 years since it first use in rehabilitation a number of models of the operation of braided pneumatic Muscle Actuator (pMA) type systems have been developed, each improving on the last. Hannaford et al considered the effects of friction within the actuator and the thickness of the rubber liner in enhancing the model [3]. Davis et al further refined the models to improve the accuracy [10]-[11]. However, in all these instances to make effective use of the 'soft' properties of these actuators, accurate control of the performance is essential, and in addition to the efforts on

* E-mail address: vassilios.tsachouridis@ieee.org ISSN: 1791-2377 (C) 2011 Kavala Institute of Technology. All rights reserved. modelling, extensive work has sought to achieve accurate control of the pMA.

One approach based on adaptive techniques was developed by [4] where the model of the system was estimated at each sample interval. Feed-forward proportional plus integral plus derivative (PID) schemes have been applied to conventional pneumatic actuators to control the position of SCARA robot [12]. More recently, [13] considered fuzzy logic to control the torque on a onelink manipulator driven by pMAs while [7] uses a neural network to control a rubbertuator arm. Simulation results from different nonlinear control schemes including back stepping, gain scheduling and sliding mode controllers using a theoretical model of the pMA were also presented recently by [14].

In this paper a new model and controller for a pMA powered joint is presented. The modelling of the joint is based on a simplified pMA force/displacement model developed by [3] which results in a theoretical model directly related to the actuator parameters. This enables the development of a new control scheme based on this theoretical model. The pressure control of the actuators is achieved by means of inexpensive on/off solenoid valves. In section 2 the simplified force generation model of the pMA is presented. Using this model section 3 illustrates the derivation of the model for a single pMA powered joint. The outcome of the model verification is presented in section 4 while the control design for the system is discussed in section 5 . The presentation of the control system design is general in order to cover any system with similar model structure. Finally, section 6 displays the simulations and experimental results from the application of the 
control scheme to the experimental joint and section 7 outlines some general conclusions and future work.

In terms of notation, we denote with 0 zero matrices of appropriate dimensions. $N, C$ and $\mathfrak{R}$ denote the sets of natural, complex and real numbers respectively. When the last sets are generalized in Euclidean spaces the corresponding dimensions of these spaces are shown as exponents to $N, C$ and $\mathfrak{R}$. In addition real Euclidean spaces with nonnegative elements are indicated with an additional + subscript. Vectors and matrices are denoted in lower and upper case respectively, with bold italic type characters. Scalars are denoted in upper or lower case single italic type characters, but in the figures they are denoted with normal (not italic) type characters. The rest of the notation is standard to the control systems literature.

\section{Modelling of pMA}

Two main approaches to the force generation problem have been developed by researchers, with the first based on energy modelling [3], and the second using force profiles of the surface pressure [4]-[5]. In developing the new model to be presented in this work the second of these approaches will be used. However, both methods provide the same base model and therefore a mixture of techniques is feasible. Figure 1a presents the geometry of the actuator structure. The actuator is considered to have a cylindrical shape. The length and the diameter of the cylinder $L$ and $D$ respectively can be expressed as functions of the muscle's braided shell parameters as shown in the braided structure in Figure 1a. These are: (i) the length of one thread of the braided structure $b$, (ii) the number of turns of one thread $n$ and (iii) the braid interweave angle $\theta$.

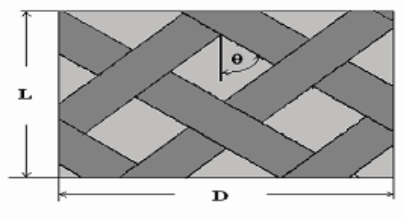

(a)

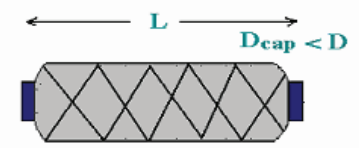

(c)

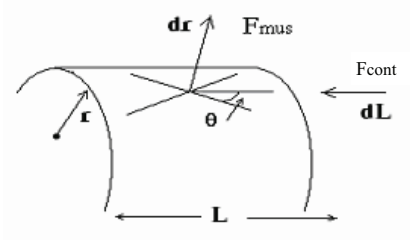

(e)

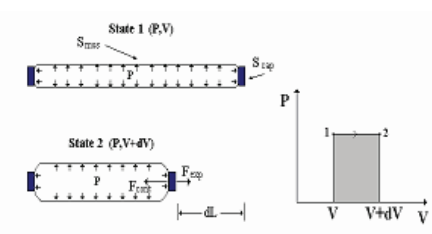

(b)

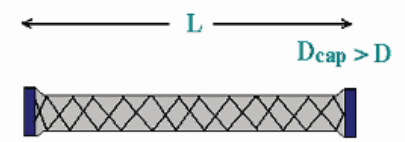

(d)

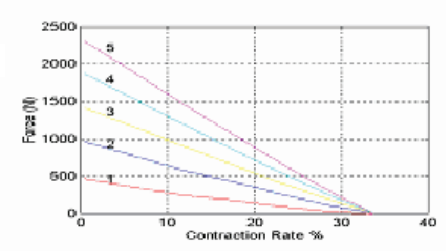

(f)
Figure 1 (a) Geometry of pneumatic muscle actuator. (b) Actuator transition states. (c) (Case I) End-Cap diameter smaller than muscle diameter $D$. (d) (Case II) End-Cap diameter greater than muscle diameter $D$. (e) Radial and contractile force relation. (f) Experimental Force/Displacement profiles

\section{$L$ and $D$ are given by}

$L=b \cos (\theta)$

and

$$
D=\frac{b \sin (\theta)}{n \pi}
$$

To calculate the contraction force in an energised muscle the following transition from fully dilated state $1(P, V)$ to partially contracted state $2(P, V+d V)$ is considered, Figure $1 \mathrm{~b}$.

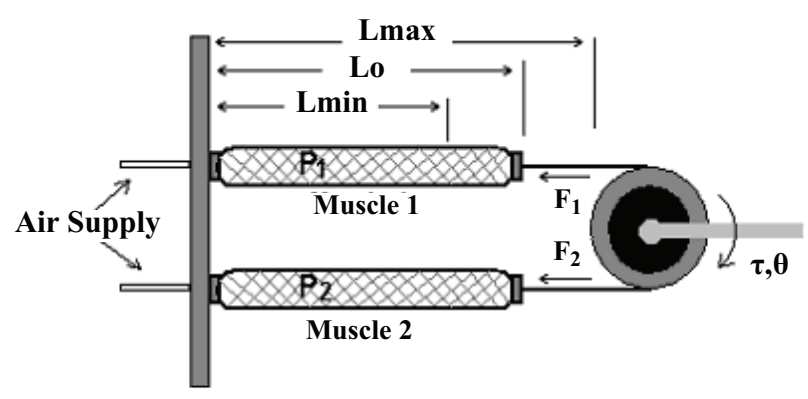

(a)

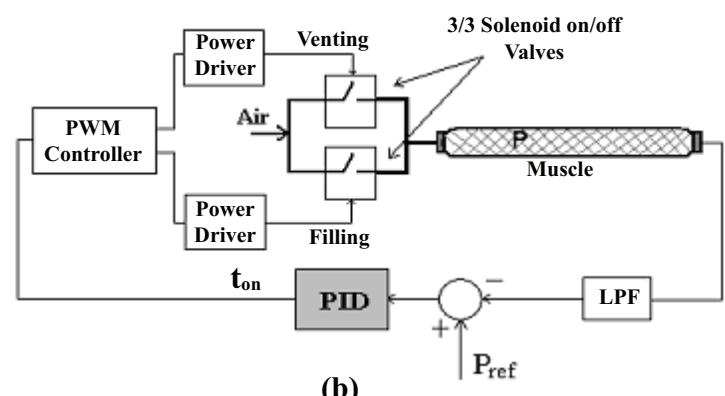

(b)

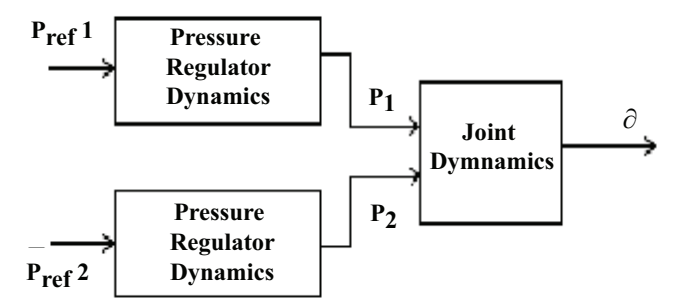

(c)

Figure 2 (a) Schematic of the experimental joint. (b) Control scheme of the muscle pressure. (c) Schematic representation of the joint model

The pressure inside the pMA is considered to remain constant during the transition (isobaric operation). The pneumatic energy stored during the transition can be obtained from the surface below the transition curve shown in the $(P, V)$ diagram in Figure $1 \mathrm{~b}$ and is equal to $E_{p}=P d V$. This input pneumatic energy induces two force components as shown in Figure 1b. These are a contractile force component $F_{\text {cont }}$ produced by pressure on the sidewalls of the pMA, and an expansion force component $F_{\text {exp }}$ created by the pressure on the end cap surface. 
$F_{\text {cont }}=a P S_{\text {mus }}$

$F_{\exp }=P S_{\text {cap }}$

Where $S_{m u s}$ is the muscle body surface area, $S_{c a p}$ is the end cap surface area and $\alpha$ is a conversion factor between the radial force induced by the pressure on the muscle body surface and the resultant contractile force. Therefore, the actual driving force is given by

$$
F=F_{\text {cont }}-F_{\text {exp }}
$$

Considering first an ideal cylinder with an end cap diameter that is small compared to the diameter of the muscle Figure 1c. Then the muscle wall surface area and the cap surface area are given by:

$S_{\text {mus }}=\pi D L=\frac{b^{2} \sin (\theta) \cos (\theta)}{n}$

$S_{\text {cap }}=\frac{\pi D^{2}}{4}=\frac{b^{2} \sin ^{2}(\theta)}{4 n^{2} \pi}$

if $D=D_{o} \sin (\theta)>D_{\text {cap }}$ (i.e. $\theta>\sin ^{-1}\left(\frac{D_{\text {cap }}}{D_{o}}\right)$ ), where $\theta$ is the braid interweave angle, $D$ is the muscle diameter, $D_{O}$ is the maximum theoretical muscle diameter at a braid interweave angle of $\theta=90^{\circ}, D_{\text {cap }}$ is the end cap diameter, and $L$ is the length of the muscle as defined earlier. When the cap diameter $D_{\text {cap }}$ is greater than the muscle diameter as may be the case near full dilation especially for thin muscles, Figure 1d, then $\theta<\sin ^{-1}\left(\frac{D_{c a p}}{D_{o}} \vartheta\right)$, the $S_{c a p}$ surface will be given by

$$
S_{c a p}=\frac{\pi D_{c a p}^{2}}{4}
$$

To determine the actual actuator force the conversion factor $\alpha$ must be calculated. This is achieved using the conservation principle between the work done by the radial force and the work done by the contractile force, Figure 1e. According to the conservation principle the mechanical work done during the radial displacement of the muscle $d D$ is equal to the mechanical work undertaken during the linear displacement of the muscle along the muscle axis $d L$.

The work produced by the radial force $F_{m u s}$ is given by

$$
d W_{\text {mus }}=F_{m u s} d r
$$

where $r$ is the radius of the muscle. The work along the muscle axis is given by

$d W_{\text {cont }}=F_{\text {cont }}(-d L)$
Assuming work conservation

$$
F_{\text {cont }}=\frac{d D}{-2 d L} F_{\text {mus }}=a F_{\text {mus }}
$$

Hence,

$$
a=\frac{d D}{-2 d L}=\frac{1}{2 n \pi \tan (\theta)}
$$

Combining (12), (3), (4) and (5) we get the formula for the driving force as $F=\frac{P b^{2} \cos ^{2}(\theta)}{2 n^{2} \pi}-\frac{P b^{2} \sin ^{2}(\theta)}{4 n^{2} \pi}$. Therefore, if $\theta>\sin ^{-1}\left(\frac{D_{c a p}}{D_{o}}\right)$

$$
F=\frac{\pi D_{o}^{2} P}{4}\left(3 \cos ^{2}(\theta)-1\right)
$$

In the case that the end-cap diameter is bigger than the muscle diameter, if $\theta<\sin ^{-1}\left(\frac{D_{c a p}}{D_{O}}\right),(13)$ is modified [10] to

$F=\frac{\pi P}{4}\left(2 D_{o}^{2} \cos ^{2}(\theta)-D_{c a p}^{2}\right)$

where $D_{O}$ is maximum theoretical muscle diameter at a braid interweave angle of $\theta=90^{\circ}$ and $P$ is the operating pressure. Details on how to derive (14) can be found in [3], [10]. The force equation (14) for $\theta>\sin ^{-1}\left(\frac{D_{c a p}}{D_{O}}\right)$ is identical with the one obtained by [3]. However, the equation in [3] does not consider the surface area of the end-cap, which can result in a reduction of the driving force when the actuator approaches its maximum length. Further refinements can improve the accuracy of the above force equation by incorporating the liner thickness effect observed by [3], considering the muscle shape as a cylinder with curved end-parts [10] and the change on the length of the thread of the braided structure [11]. However, these extra improvements are not considered in this work to make the modelling process practicable and do not significantly effect the accuracy of the models. Equation (13) can also be written as a function of the muscle length $L$ and the length of one thread of the braided structure $b$ as follows.

$F=\frac{\pi D_{o}^{2} P\left(3 L^{2}-b^{2}\right)}{4 b^{2}}$

Simulated results using the above equation and experimental data, Figure 1f, for different sizes of muscles suggest that the pneumatic muscle behaves like a variable compliance spring system. Based on this observation and considering constant pressure due to the small length change $d L$, the muscle spring stiffness per unit length can be computed from (15) 
$K=\frac{d F}{d L}=\frac{6 \pi D_{O}^{2} P L}{4 b^{2}}$

As it can be seen from (16) the stiffness of the muscle is a function of the operating pressure $P$ and the length of the muscle $L$. We can now calculate the stiffness per unit length and pressure $K_{p r}$ from (17).

$K_{p r}=\frac{d K}{d P}=\frac{6 \pi D_{O}^{2} L}{4 b^{2}}=K_{g a s} L$

The muscle force equation can now be rewritten as shown below to replicate a spring force equation.

$$
F=K_{p r} P\left(L-L_{\text {min }}\right)
$$

where $L_{\min }$ is the length of the muscle when it is fully contracted. Equation (18) is the form of the model developed by [3] and represents the muscle as a nonlinear quadratic spring with unknown elastic spring constant. As it can be seen in our case the spring elastic constant can be expressed as a function of the muscle parameters simplifying the modelling process. The muscle force equation in the form presented above will be used for the modelling of the experimental apparatus described in the next section.

\section{Modelling an Antagonistic Muscle Actuated Joint}

Based on the static force equation of the pMA the state space model of an experimental joint apparatus powered by two antagonistic muscles can be computed, Figure 2a. In figure 2a, $L_{\min }$ is the length of the muscle when it is fully contracted, $L_{O}=L_{\min }+\frac{L_{\max }-L_{\min }}{2}$ is the initial dilated length of muscle which is equal to the half of the maximum muscle displacement in order to maximise the range of motion of the joint, $L_{\text {max }}$ is the maximum dilated length, $r$ is the radius of the pulley and $P_{1}, P_{2}$ are the gauge pressures inside the two muscles. The equation that describes the motion of the joint is given by

$J \ddot{\theta}+D \dot{\theta}=\tau$

where $J, D, \tau$ denote the joint inertia, damping and torque respectively. Considering now the antagonistic pair of muscles shown in Figure 2a and using the muscle force equation (18), the forces developed are given by:

$$
\begin{aligned}
& F_{1}=K_{p r} P_{1}\left(L_{o}-L_{\text {min }}+r \theta\right) \\
& F_{2}=K_{p r} P_{2}\left(L_{o}-L_{\text {min }}-r \theta\right)
\end{aligned}
$$

Equations (20) and (21) can be rewritten as:

$$
\begin{aligned}
& F_{1}=K_{p r} P_{1}\left(\Delta L_{o}+r \theta\right)=K_{\text {gas }} P_{1} L_{1} \Delta L_{1} \\
& F_{2}=K_{p r} P_{2}\left(\Delta L_{o}+r \theta\right)=K_{\text {gas }} P_{2} L_{2} \Delta L_{2}
\end{aligned}
$$

Where $\Delta L_{O}=L_{O}-L_{\text {min }}=\frac{L_{\max }-L_{\min }}{2}$ is the initial dilation for the muscles and $L_{1}=L_{\text {min }}+\Delta L_{1}, L_{2}=L_{\text {min }}+\Delta L_{2}$, are the length of the muscles at any joint positions. The dilations for the two muscles at any joint position are $\Delta L_{1}=\Delta L_{O}+r \theta$ and $\Delta L_{1}=\Delta L_{O}-r \theta$. Using (22) and (23) the torque developed at the joint can now be computed:

$$
\begin{aligned}
& T=\left(F_{2}-F_{1}\right) r= \\
& =\left(K_{\text {gas }} P_{2} L_{2} \Delta L_{2}-K_{\text {gas }} P_{1} L_{1} \Delta L_{1}\right) r
\end{aligned}
$$

The equation of motion of the single joint in Figure $2 b$ can be written as:

$$
\begin{aligned}
& \ddot{\theta}=\frac{-D}{J} \dot{\theta}+\frac{K_{\text {gas }} r^{3}\left(P_{2}-P_{1}\right)}{J} \theta^{2}- \\
& -\frac{K_{\text {gas }} r^{2}\left(P_{2}+P_{1}\right)\left(2 \Delta L_{o}+L_{\text {min }}\right)}{J} \theta+ \\
& +\frac{K_{\text {gas }} r\left(P_{2}-P_{1}\right) \Delta L_{o}\left(L_{\text {min }}+\Delta L_{o}\right)}{J}
\end{aligned}
$$

By selecting the states of the system to be $\mathrm{x}_{1}=\theta$ and $\mathrm{x}_{2}=\dot{\theta}$, the state equations of the system can be formulated from (25) as

$$
\begin{aligned}
& {\left[\begin{array}{c}
\dot{x}_{1} \\
\dot{x}_{2}
\end{array}\right]=\left[\begin{array}{ccc}
0 & 1 \\
\frac{K_{g a s} r^{3}\left(P_{2}-P_{1}\right)}{J} x_{l}-\frac{K_{g a s} r^{2}\left(P_{1}+P_{2}\right) L_{\text {max }}}{J} & -\frac{D}{J}
\end{array}\right] *} \\
& *\left[\begin{array}{c}
x_{1} \\
x_{2}
\end{array}\right]+\left[\begin{array}{c}
0 \\
\frac{K_{g a s} r\left(L_{\text {max }}^{2}-L_{\text {min }}^{2}\right)}{4 J}
\end{array}\right]\left[P_{2}-P_{1}\right]
\end{aligned}
$$

The above joint model describes the joint dynamics but since it is based on the static force equation of a pMA it does not incorporate the dynamics of the actuator air pressures $P_{1}$, $P_{2}$. In this system the air pressure inside each of the two muscles is regulated using on/off solenoid 3/3 valves (MAKE), Figure $2 b$.

As can be seen from Figure 2b, a PID controller is used to calculate the on/off times of the venting/filling valves and effectively regulates the pressure. The dynamics of each of the two pressure regulators for $P_{1}, P_{2}$ were identified in MATLAB using experimental input/output pressure data from each muscle. A schematic representation of the overall joint dynamic model is shown in Figure 2c.

\section{Model Verification}

To verify the model developed in the previous sections, the experimental joint was interfaced with the control system illustrated in Figure $3 \mathrm{a}$ and the outcome of the model is compared with the results from the experimental setups. 


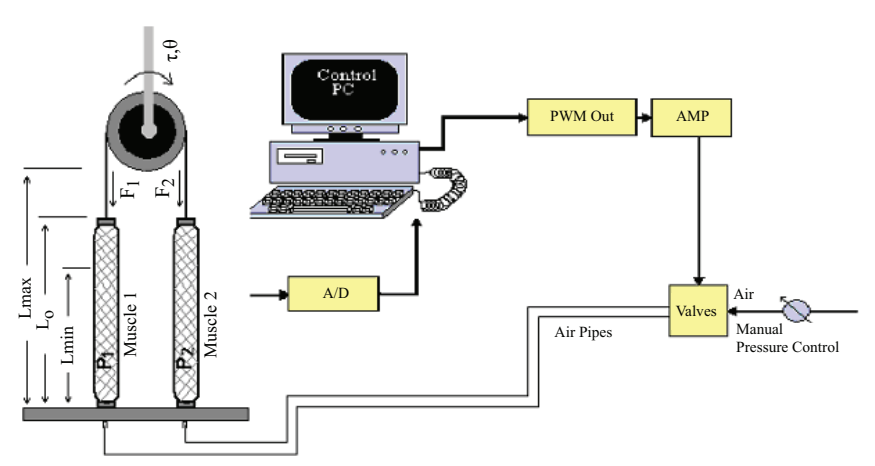

(a)

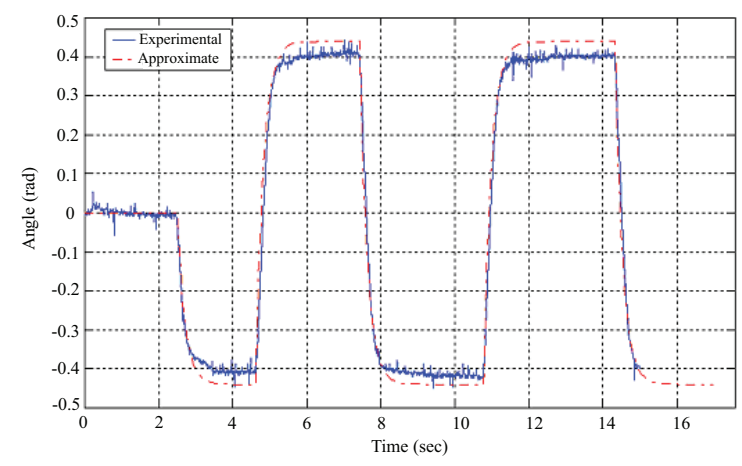

(b)

Figure 3. (a) Schematic diagram of the air control system. (b) Experimental and theoretical step response

The parameters of the experimental system are: $b=0.23$ $(\mathrm{m}), D=0.028, K_{\text {gas }}=0.0509(\mathrm{~N} / \mathrm{m} / \mathrm{Pa}), L_{\text {min }}=0.145(\mathrm{~m})$, $L_{\text {max }}=0.205(\mathrm{~m}), r=0.03(\mathrm{~m}), J=8.125 \times 10^{-6}\left(\mathrm{Kgm}^{2}\right)$.

In the above system the air pressure is controlled using pulse width modulation with a switching frequency up to 300 $\mathrm{Hz}$ and a fast response time of less than $2 \mathrm{~ms}$. By varying the duty cycle of the PWM, regulation of the airflow can be achieved, and thus the amount of muscle contraction can be controlled. The system uses a Pentium III (1GHz) based PC to produce an interrupt driven PWM signal with appropriate data acquisition card and amplifiers to drive the solenoid valves.

The open loop step response of the system to the input pressure variation $\Delta P=P_{r e f_{2}}-P_{r e f_{1}}$ was recorded. The two pressure references $P_{\text {ref }}, P_{\text {ref }}$, were set as

$$
\left(P_{r e f_{1}}, P_{r e f_{2}}\right)=\left(\frac{P_{\max }-\Delta P}{2}, \frac{P_{\max }+\Delta P}{2}\right)
$$

with $P_{\max }=400000(\mathrm{~Pa})$. This gives a balanced input pressure variation and the system can be considered as a SISO system. The same generated $\Delta P \in[-100000,100000](\mathrm{Pa})$ profile was used as input for the theoretical system model, and the theoretical step response generated by MATLAB was plotted against the experimental one in Figure $3 b$. Figure $3 b$ shows a good transient equivalence between the theoretical and the experimental responses although, a steady state error between the two responses can be observed which is believed to be due to the nonmodelled system parameters mentioned previously.

\section{Control system design}

As can be seen from equation (26) the system's nonlinear model can be considered as special case of the nonlinear dynamical system

$$
\begin{aligned}
& \dot{x}(t):=A x(t)+(B+g(y(t), t)) u(t)+w(t), \\
& x(0):=x_{o} \\
& y(t):=C x(t)
\end{aligned}
$$

where, $x(t) \in \mathfrak{R}^{n}, u(t) \in \mathfrak{R}^{m}, y(t) \in \mathfrak{R}^{m}$, are defined as the system's state, input and output respectively and $A \in \mathfrak{R}^{n \times n}, B \in \mathfrak{R}^{n \times m}$, $C \in \mathfrak{R}^{m \times n}$, are the corresponding constant matrix coefficients of the above vectors. $w(t) \in \mathfrak{R}^{n}$ represent unknown system dynamics due to model uncertainties and disturbances. $t \in \mathfrak{R}_{+}$is the continuous time variable and $g(y(t), t) \in \mathfrak{R}^{m}$ is a nonlinear function of the system's output and time.

System (28), (29) with sampling $t_{S} \in \mathfrak{R}_{+}-\{0\}$, according to the Nyquist sampling theorem [18], can be approximated as

$$
\begin{aligned}
& x\left(k t_{s}+t_{s}\right):=A_{d} x\left(k t_{s}\right)+\quad k \in N, x(0):=x_{o} \\
& +\left(B_{d}+g_{d}\left(y\left(k t_{s}\right), k t_{s}\right)\right) u\left(k t_{s}\right)+w\left(k t_{s}\right)
\end{aligned}
$$

$y\left(k t_{s}\right):=C x\left(k t_{s}\right)$

where

$A_{d}:=e^{A t_{s}}$

$B_{d}:=\left(\int_{0}^{t_{s}} e^{A \tau} d \tau\right) B=-\left(I_{n}-A_{d}\right) A^{-1} B$

$g_{d}\left(y\left(k t_{s}\right), k t_{s}\right):=\left(\int_{0}^{t_{s}} e^{A \tau} d \tau\right) g\left(y\left(k t_{s}\right), k t_{s}\right)=$

$=-\left(I_{n}-A_{d}\right) A^{-1} g\left(y\left(k t_{s}\right), k t_{s}\right)$

Hence, we have the next result.

\section{Theorem 1 (Tracking Problem);}

Consider the system (30), (31) subject to (32)-(34) and suppose that $(A, B)$ is controllable, $A$ is asymptotically stable, $(A, B, C)$ has no system zeros at the origin and $\operatorname{rank}(C B)=m$. Furthermore, suppose that $\forall t \in \mathfrak{R}_{+}, w(t)|\leq \bar{w} \geq 0| g,(y(t), t) \mid \leq \bar{g} \geq 0$, $\left(B+g(y(t), t)\left(B+g(y(t), t)^{\#} B=B\right.\right.$, where $\#$ denotes the generalised inverse of a matrix [15], and $\left|y_{r}(t)\right| \leq \bar{y}_{r} \geq 0$, where $y_{r}(t) \in \mathfrak{R}^{m}$. If $\lim _{k \rightarrow \infty}\left(y_{r}\left(k t_{s}\right)\right)$ and $\lim _{k \rightarrow \infty}\left(t_{s}^{-1}\left(w\left(k t_{s}+t_{s}\right)-w\left(k t_{s}\right)\right)\right)$ exist, then

$$
\begin{aligned}
& \lim _{k \rightarrow \infty}\left(y\left(k t_{s}\right)\right)=\lim _{k \rightarrow \infty}\left(y_{r}\left(k t_{s}\right)\right)-K_{i n}^{-1} C\left(I_{n}-A_{d}\right)^{-1} * \\
& * \lim _{k \rightarrow \infty}\left(t_{s}^{-1}\left(w\left(k t_{s}+t_{s}\right)-w\left(k t_{s}\right)\right)\right)
\end{aligned}
$$


subject to

$u\left(k t_{s}\right):=-\left(B+g\left(y\left(k t_{s}\right), k t_{s}\right)\right)^{\#} B\left(C A^{-1} B\right)^{-1} *$

$*\left(K_{p}\left(y\left(k t_{s}\right)-y_{r}\left(k t_{s}\right)\right)+t_{s} K_{i n} \sum_{i=0}^{k}\left(y\left(i t_{s}\right)-y_{r}\left(i t_{s}\right)\right)-y_{r}\left(k t_{s}\right)\right)$

where

$K_{p:=\operatorname{diag}}\left(\left[K_{p_{1}} \cdots K_{p_{m}}\right]\right)$

$K_{\text {in }:=\operatorname{diag}}\left(\left[\begin{array}{lll}K_{i n_{1}} & \cdots & K_{i n_{m}}\end{array}\right]\right)$

$K_{p} \in \mathfrak{R}_{+}^{m \times m}, K_{\text {in }} \in \mathfrak{R}_{+}^{m \times m}$ are design parameters and $\operatorname{rank}\left(K_{\text {in }}\right)$ $=m$.

Proof.

The controllability of $(A, B)$ is technical and implies the existence of a control law $u(\mathrm{t})$ for the control of (28), (29) and consequently for the control of (30), (31). First we prove that rank $\left(C A^{-1} B\right)=m$ (i.e. $\left(C A^{-1} B\right)^{-1}$ in (36) exists). The asymptotic stability of $A$ constraints its eigenvalues $\lambda i(A), i=1,2, \ldots, n$ to have strictly negative real parts and this implies $\operatorname{rank}(A)=n, \operatorname{det}(A)$ $\neq 0$ and therefore the existence of $A^{-1}$. Now, the system zeros of the triplet $(A, B, C)$ are defined as $\operatorname{det}\left(\left[\begin{array}{cc}s I_{n}-A & B \\ C & 0\end{array}\right]\right)=0, s \in \mathrm{C}$ [16]. Since $(A, B, C)$ has no system zeros at the origin and det $(A) \neq 0$ we have equivalently

$\left.\operatorname{det}\left(\left[\begin{array}{cc}s I-A & B \\ C & 0\end{array}\right]\right)\right)_{s=0} \neq 0 \Leftrightarrow \operatorname{det}(-A) \operatorname{det}\left(C(-A)^{-1} B\right) \neq 0 \Leftrightarrow$ $\Leftrightarrow \operatorname{det}\left(C A^{-1} B\right) \neq 0 \Leftrightarrow \operatorname{rank}\left(C A^{-1} B\right)=m$

In addition, because $A$ is asymptotically stable we have consequently $\left|\lambda_{i}\left(A_{d}\right)\right|=e^{t_{s} \operatorname{Re}\left(\lambda_{i}(A)\right)}<1,-1<R_{e}\left(\lambda_{i}\left(A_{d}\right)\right)<1$, $\lambda_{i}\left(I_{n}-A_{d}\right)=1-\lambda_{i}\left(A_{d}\right) \neq 0$ for $i=1,2, \ldots, n$ and therefore rank $\left(I_{n}-A_{d}\right)=n$ which implies that $\left(I_{n}-A_{d}\right)^{-1}$ exists in (33), (34)

Let

$\tilde{u}\left(k t_{s}\right):=\left(C A^{-1} B\right)^{-1}\left(\begin{array}{l}K_{p}\left(y\left(k t_{s}\right)-y_{r}\left(k t_{s}\right)\right)+ \\ +t_{s} K_{i n} \sum_{i=0}^{k}\left(y\left(i t_{s}\right)-y_{r}\left(i t_{s}\right)\right)-y_{r}\left(k t_{s}\right)\end{array}\right)$

Substituting (36) into (30) with respect to (32)-(34), (37)(39) and because $\left(B+g\left(y\left(k t_{S}\right), k t_{S}\right)\left(B+g\left(y\left(k t_{S}\right), k t_{S}\right){ }^{\#} B=B\right.\right.$, $\forall k \in N$, we have

$$
\begin{aligned}
& x\left(k t_{s}+t_{s}\right)=A_{d} x\left(k t_{s}\right)+B_{d} \tilde{u}\left(k t_{s}\right)+w\left(k t_{s}\right)= \\
= & A_{d} x\left(k t_{s}\right)-\left(I_{n}-A_{d}\right) A^{-1} B \tilde{u}\left(k t_{s}\right)+w\left(k t_{s}\right)
\end{aligned}
$$

Also let

$G_{d}(z):=C\left(z I_{n}-A_{d}\right)^{-1} B_{d}=$

$=-C\left(z I_{n}-A_{d}\right)^{-1}\left(I_{n}-A_{d}\right) A^{-1} B{ }^{, z \in \mathrm{C}}$
In view of (41) and (30), (31) can be written in the Ztransform domain [18] as

$y(z)=G_{d}(z) \widetilde{u}(z)+C\left(z I_{n}-A_{d}\right)^{-1} w(z)+C x(0)$

where $\mathrm{z} \in \mathrm{C}$ is the Z-transform variable [18] and $\bar{u}(z)$ is the Z-transform of (39) given by

$$
\begin{aligned}
& \tilde{u}(z)=\left(C A^{-1} B\right)^{-1}(z-1)^{-1} * \\
& *\left((z-1)\left(K_{p}+I_{m}\right)+t_{s} K_{\text {in }}\right)\left(y(z)-y_{r}(z)\right)
\end{aligned}
$$

After substituting (43) and (41) in (42) and rearranging common factors and multiplying both sides with $\mathrm{z}^{-1}(\mathrm{z}-1)^{2}$ we get

$$
\begin{aligned}
& \left(\begin{array}{l}
(z-1) I_{n}+C\left(z I_{n}-A_{d}\right)^{-1}\left(I_{n}-A_{d}\right) A^{-1} B\left(C A^{-1} B\right)^{-1} * \\
*\left((z-1)\left(K_{p}+I_{m}\right)+t_{s} K_{i n}\right)
\end{array}\right) z^{-1}(z-1) y(z) \\
& =\left(\begin{array}{l}
C\left(z I_{n}-A_{d}\right)^{-1}\left(I_{n}-A_{d}\right) A^{-1} B\left(C A^{-1} B\right)^{-1} * \\
*\left((z-1)\left(K_{p}+I_{m}\right)+t_{s} K_{i n}\right)
\end{array}\right) z^{-1}(z-1) y_{r}(z) \\
& +C\left(z I_{n}-A_{d}\right)^{-1} z^{-1}(z-1)^{2} w(z) .
\end{aligned}
$$

Because $\forall k \in N,\left|w\left(k t_{S}\right)\right| \leq \bar{w} \geq 0,\left|g\left(y\left(k t_{S}\right), k t_{S}\right)\right| \leq$ $\bar{g} \geq 0$, and $\left|y_{r}\left(k t_{S}\right)\right| \leq \bar{y}_{r} \geq 0$, it follows that $u\left(k t_{S}\right)$ in (36) is bounded and so are $x\left(k t_{S}\right)$ and $y\left(k t_{S}\right)$. Therefore, since $\lim _{k \rightarrow \infty}\left(y_{r}\left(k t_{s}\right)\right)$ and $\lim _{k \rightarrow \infty}\left(t_{s}^{-1}\left(w\left(k t_{s}+t_{s}\right)-w\left(k t_{s}\right)\right)\right)$ exist by assumption, it follows that $\lim _{k \rightarrow \infty}\left(x\left(k t_{s}\right)\right) \neq \pm \infty, \lim _{k \rightarrow \infty}\left(y\left(k t_{s}\right)\right) \neq \pm \infty$, $\lim _{k \rightarrow \infty}\left(y_{r}\left(k t_{s}\right)\right) \neq \pm \infty$ and $\lim _{k \rightarrow \infty}\left(w\left(k t_{s}+t_{s}\right)-w\left(k t_{s}\right)\right) \neq \pm \infty$. From the initial value theorem the last results equivalently imply that $\lim _{z \rightarrow 1}\left(z^{-1}(z-1) x(z)\right) \neq \pm \infty, \lim _{z \rightarrow 1}\left(z^{-1}(z-1) y(z)\right) \neq \pm \infty$ and $\lim _{z \rightarrow 1}\left(z^{-1}(z-1)^{2} w(z)\right) \neq \pm \infty$. Hence, considering the $\lim _{z \rightarrow 1}((44))$ and after simplifying terms we finally get

$$
\begin{aligned}
& t_{s} K_{i n} \lim _{z \rightarrow 1}\left(z^{-1}(z-1) y(z)\right)= \\
& =t_{s} K_{i n} \lim _{z \rightarrow 1}\left(z^{-1}(z-1) y_{r}(z)\right)-C\left(I_{n}-A_{d}\right)^{-1} \lim _{z \rightarrow 1}\left(z^{-1}(z-1)^{2} w(z)\right) \\
& \Rightarrow t_{s} K_{i n} \lim _{k \rightarrow \infty}\left(y\left(k t_{s}\right)\right)= \\
& =t_{s} K_{i n} \lim _{k \rightarrow \infty}\left(y_{r}\left(k t_{s}\right)\right)-C\left(I_{n}-A_{d}\right)^{-1} \lim _{k \rightarrow \infty}\left(w\left(k t_{s}+t_{s}\right)-w\left(k t_{s}\right)\right) \\
& \Rightarrow \lim _{k \rightarrow \infty}\left(y\left(k t_{s}\right)\right)= \\
& =\lim _{k \rightarrow \infty}\left(y_{r}\left(k t_{s}\right)\right)-K_{i n}^{-1} C\left(I_{n}-A_{d}\right)^{-1} \lim _{k \rightarrow \infty}\left(t_{s}^{-1}\left(w\left(k t_{s}+t_{s}\right)-w\left(k t_{s}\right)\right)\right)
\end{aligned}
$$


Remark 1;

The main philosophy behind Theorem 1 is the explicit and exact cancellation of the nonlinearity $g_{d}\left(y\left(k t_{S}\right), t_{S}\right)$ in $(30),(31)$. Note that because of (39), system (30), (31) is reduced to a linear system (namely (40) and (42)), with the transfer function (41). In theory any linear control system design can be applied to (40), (42) in order to solve the tracking problem. Having simplicity as one of the priorities in the overall assembly of our system, the first prototype we present here is a very simple and robust controller which consists of a well-tuned PI controller with a feed forward action. Note that the presence of $-C A^{-1} B$ in (36) and (39) is not accidental since that is the DC gain of (41). In practice this often eases the selection of $K_{p}$ and $K_{\text {in }}$ during the design for which the individual elements have values in the range $(0,10)$. This is a major advantage compared to blind tuning of the PI and the tuning of other possible controllers for (41). A block diagram of the overall control system based on (36) is shown in Figure 4, where $\mathrm{ZOH}$ blocks denote both zero-order-hold and sampling.

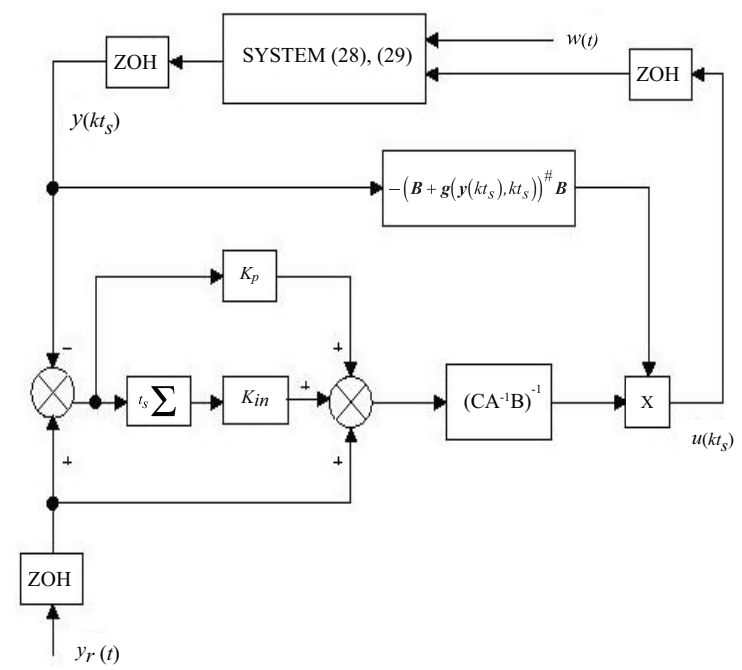

Figure 4. Sampled data control system

\section{Remark 2;}

If $w(t) \in \mathfrak{R}^{n}$ is constant $\forall t \in \mathfrak{R}_{+}$then $w\left(k t_{s}+t_{S}\right)-w\left(k t_{S}\right)=0$,

$\forall k \in N$ and therefore (35) is simplified to $\lim _{k \rightarrow \infty}\left(y\left(k t_{s}\right)\right)=$ $\lim _{k \rightarrow \infty}\left(y_{r}\left(k t_{s}\right)\right) \cdot K_{i n}^{-1} C\left(I_{n}-A_{d}\right)^{-1} \lim _{k \rightarrow \infty}\left(t_{s}^{-1}\left(w\left(k t_{s}+t_{s}\right)-w\left(k t_{s}\right)\right)\right)$, which means that we have perfect tracking of the reference signal $y_{r}\left(k t_{s}\right)$. Moreover, if $\lim _{k \rightarrow \infty}\left(y_{r}\left(k t_{s}\right)\right)$ or $\lim _{k \rightarrow \infty}\left(t_{s}^{-1}\left(w\left(k t_{s}+t_{s}\right)-w\left(k t_{s}\right)\right)\right)$ does not exist (e.g. $y\left(k t_{S}\right)$ or $w\left(k t_{S}\right)$ is a periodic function), then perfect tracking is not possible. Nevertheless, it will be shown later via simulations that in cases where $\lim _{k \rightarrow \infty}\left(y_{r}\left(k t_{s}\right)\right)$ or $\lim _{k \rightarrow \infty}\left(t_{s}^{-1}\left(w\left(k t_{s}+t_{s}\right)-w\left(k t_{S}\right)\right)\right)$ does not exist, $y\left(k t_{S}\right)$ can still follow $y_{r}\left(k t_{S}\right)$ with a delay. Also, depending on the dynamics of the system it is possible to significantly reduce that delay, so that in practical applications it can be considered as null.

\section{Remark 3;}

The above analysis assumes that the systems (28), (29) and (30), (31) are deterministic. The case of the last systems being stochastic (e.g. by considering measurement random noise and disturbances), complicates the mathematics of the problem tremendously even if this is posed as a stochastic control problem with respect to the expected values of the system's stochastic variables. The reason for this is the nonlinearity $g_{d}\left(y\left(k t_{S}\right), t_{S}\right)$, where $y\left(k t_{S}\right)$ is now a stochastic variable. Because the effect of randomness in our system is very small and practically negligible, the stochastic analysis of our problem is beyond the scope of this paper and is left for future research.

\subsection{Closed loop system response}

In this section we examine the response of the (30), (31) with respect to (36) and we derive norm-wise bound estimates for the control signal. This is useful to determine a priori the maximum possible magnitude of the control signal energy so that the system's output and input lie within specified limits due to physical or any other kind of limitations. Although the above bounds can be very conservative in the general case of multiple input multiple output (MIMO) tracking systems, this conservatism is not the case for single input single output (SISO) tracking systems. Therefore, we could have an additional analysis tool to check if for the selected $K_{p} \in \mathfrak{R}_{+}^{m \times m}$ and $K_{i n} \in \mathfrak{R}_{+}^{m \times m}$ in (37) and (38), system input and output saturation are avoided. Because of the asymptotically stable $A$ and bounded $w\left(k t_{S}\right)$ and $y_{r}\left(k t_{S}\right)$, if $y\left(k t_{S}\right)$ and $u\left(k t_{S}\right)$ are within their defined limits, then obviously the exponential stability of the overall system can be guaranteed since no saturation nonlinearities will take place.

Equations (30), (31), (36) and (39), if augmented together, form the closed loop system (45), (46).

$$
\begin{aligned}
& x_{R}\left(k t_{s}+t_{s}\right):=A_{d_{R}} x\left(k t_{s}\right)+B_{d_{R}} u_{R}\left(k t_{s}\right)+w_{R}\left(k t_{s}\right), \\
& k \in N, \quad x_{R}(0):=x_{R_{O}} \\
& y_{R}\left(k t_{s}\right):=C_{d_{R}} x_{R}\left(k t_{s}\right) \\
& \text { where } x_{R}\left(k t_{S}\right):=\left[\begin{array}{l}
x\left(k t_{s}\right) \\
\tilde{u}\left(k t_{s}\right)
\end{array}\right], u_{R}\left(k t_{s}\right):=\left[\begin{array}{c}
y_{r}\left(k t_{s}+t_{S}\right) \\
y_{r}\left(k t_{s}\right)
\end{array}\right] \text {, } \\
& w_{R}\left(k t_{s}\right):=\left[\begin{array}{c}
w\left(k t_{s}\right) \\
0
\end{array}\right], \quad A_{d_{R}}:=E_{d}^{-1} \widetilde{A}_{d}, \\
& B_{d_{R}}:=E_{d}^{-1} \widetilde{B}_{d}, E_{d}:=\left[\begin{array}{cc}
I_{n} & 0 \\
-\left(C A^{-1} B\right)^{-1}\left(K_{p}+t_{s} K_{i n}\right) K_{p} C & I_{m}
\end{array}\right], \\
& E_{d}^{-1}:=\left[\begin{array}{cc}
I_{n} & 0 \\
\left(C A^{-1} B\right)^{-1}\left(K_{p}+t_{s} K_{i n}\right) K_{p} C & I_{m}
\end{array}\right],
\end{aligned}
$$

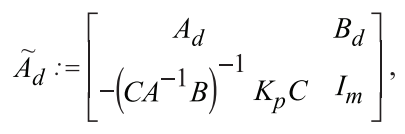

$$
\begin{aligned}
& \widetilde{B}_{d}:=\left[\begin{array}{cc}
0 & 0 \\
-\left(C A^{-1} B\right)^{-1}\left(K_{p}+t_{s} K_{i n}+I_{m}\right) & \left(C A^{-1} B\right)^{-1}\left(K_{p}+I_{m}\right)
\end{array}\right],
\end{aligned}
$$


$C_{R}:=\left[\begin{array}{cc}C & 0 \\ 0 & I_{m}\end{array}\right] \cdot$ It is apparent from (45) that we are considering as system inputs the reference signal to be tracked and its derivative, while from (46) the output vector contains the variables of interest: the system's original output and part of the control input (36) (i.e. (39)).

Now, suppose that $K_{p} \in \mathfrak{R}_{+}^{m \times m}$ and $K_{i n} \in \mathfrak{R}_{+}^{m \times m}$ result an asymptotically stable $A_{d_{R}}$. Moreover, let the limitations be (e.g. due to physical constraints) $y^{-} \leq y\left(k t_{S}\right) \leq y^{+}, \quad u^{-} \leq u\left(k t_{s}\right) \leq u^{+}$. From (36) and (39) we have $u\left(k t_{s}\right)=\left(B_{d}+g\left(y\left(k t_{s}\right), k t_{s}\right)\right)^{\#} B_{d} \tilde{u}\left(k t_{s}\right)$ and therefore $u^{-} \leq\left(B_{d}+g\left(y\left(k t_{s}\right), k t_{s}\right)\right)^{\#} B_{d} \tilde{u}\left(k t_{s}\right) \leq u^{+}$.

In general any bounded input signal can be sufficiently approximated as a series of combined piecewise continuous step, ramp and parabolic inputs, each defined in a finite length time horizon (see chapter 6 of [19] and chapter 1 of [17]). Although one may is to go further and restrict the above approximation by considering only step inputs (e.g. the case of $\mathrm{ZOH}$ sampling), for the sake of analysis we will consider all the three types of input signals above. Therefore, by studying the system behaviour of the above type of signals we can estimate the system's response to general input signals [19].

Hence, assume the response of an asymptotically stable system due to a step, ramp, or parabolic change in its input at time $t=t^{*}>0$. It is clear this response can be replicated from the system if the same step, ramp, or parabolic input is applied at $t=0$ with respect to the system's initial state condition $x(0)=x\left(t^{*}\right)$. In addition, if we restrict our attention to the magnitude of the system's output and pose the problem of determining the maximum norm of the system's input, so that the system input and output are confined within their specified limits, then it is apparent from the above considerations that the solution to this problem can be provided by the studies of the system's response with respect to step, ramp, or parabolic inputs at $t=0$.

In order to put the above concept into mathematical language, define the operators

$$
\begin{aligned}
& \Phi_{d_{S}}: N \rightarrow \Re^{(2 m) \times(n+m)} \\
& \Phi_{d_{S}}\left(k t_{s}\right):=-C_{d_{R}}\left(\sum_{i=0}^{k-1} A_{d_{R}}^{i}\right) \\
& \Phi_{d_{r}}: \mathfrak{R}_{+} \rightarrow \mathfrak{R}^{(2 m) \times(n+m)}, \\
& \Phi_{r}\left(k t_{s}\right):=-C_{d_{R}}\left(\sum_{i=0}^{k-1}(k-1-i) t_{s} A_{d_{R}}^{i}\right) \\
& \Phi_{d_{p}}: \mathfrak{R}_{+} \rightarrow \mathfrak{R}^{(2 m) \times(n+m)}, \\
& \Phi_{d_{p}}(t):=-C_{d_{R}}\left(\sum_{i=0}^{k-1}\left((k-1-i) t_{s}\right)^{2} A_{d_{R}}^{i}\right)
\end{aligned}
$$

Note that all the quantities in (47)-(49) are well defined under the assumption that $A_{d_{R}}$ is asymptotically stable. Furthermore, in view of (47)-(49), the operators can be defined

$$
\begin{aligned}
& \Phi_{d_{u_{R}}}: \rightarrow \Re^{(2 m) \times(2 m)}
\end{aligned}
$$

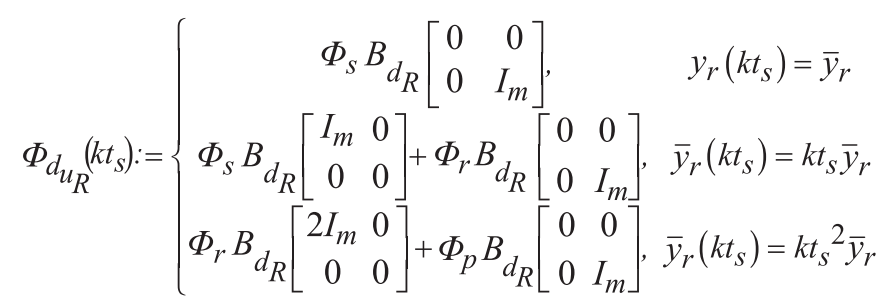

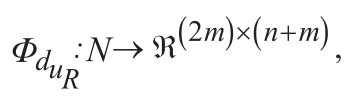

$\Phi_{w_{R_{d}}}\left(k t_{s}\right):= \begin{cases}\Phi_{d_{s},} & w_{R}\left(k t_{s}\right)=k t_{s} \bar{w}_{R} \\ \Phi_{d_{r}}, & w_{R}\left(k t_{s}\right)=k t_{s} \bar{w}_{R} \\ \Phi_{d_{p}}, & w_{R}\left(k t_{s}\right)=\left(k t_{s}\right)^{2} \bar{w}_{R}\end{cases}$

The response of (46) subject to $u_{R}\left(k t_{s}\right)=\bar{u}_{R}$, or $u_{R}\left(k t_{s}\right)=k t_{s} \bar{u}_{R}$, or $u_{R}\left(k t_{s}\right)=\left(k t_{s}\right)^{2} \bar{u}_{R}$ and $w_{R}\left(k t_{s}\right)=\bar{w}_{R}$, or $w_{R}(t)=k t_{s} \bar{w}_{R}$, or $w_{R}\left(k t_{s}\right)=\left(k t_{s}\right)^{2} \bar{w}_{R}, \bar{u}_{R}:=\left[\begin{array}{c}0 \\ \bar{y}_{r}\end{array}\right], \bar{w}_{R}:=\left[\begin{array}{c}\overline{\bar{w}} \\ 0\end{array}\right]$, where $\bar{u}_{R} \in \mathfrak{R}^{2 m},\left[\begin{array}{cc}-\infty & \\ & y_{r}^{-}\end{array}\right] \leq \bar{u}_{R} \leq\left[\begin{array}{c}+\infty U_{m} \times 1 \\ y_{r}^{+}\end{array}\right] \quad\left(U_{m} \times 1 \in N^{m}\right.$ is the unity vector full of 1 's), $\bar{y}_{r} \in \mathfrak{R}^{2 m}, \bar{y}_{r}^{-} \leq \bar{y}_{r} \leq \bar{y}_{r}^{+}$, $\bar{w}_{R} \in \Re^{n+m},-\left[\begin{array}{c}\bar{w} \\ 0\end{array}\right] \leq \bar{w}_{R} \leq\left[\begin{array}{c}\bar{w} \\ 0\end{array}\right]$, and $x_{R}(0)=x_{R_{0}}$, is given by

$y_{R}\left(k t_{s}\right)=C_{d_{R}} A_{d_{R}}^{k} x_{R}(0)+$

$+\Phi_{d_{u_{R}}}\left(k t_{s}\right) \bar{u}_{R}+\Phi_{d_{w_{R}}}\left(k t_{s}\right) \bar{w}_{R}$

where $\Phi_{d_{u_{R}}}\left(k t_{S}\right), \Phi_{d_{w_{R}}}\left(k t_{s}\right)$ are defined in (50) and (51) respectively.

The problem of finding the maximum control signal norm such that the system's output and control input do not saturate can be stated as the nonlinear optimisation problem

$$
\max \left(\left\|\left(B_{d}+g\left(y\left(k t_{s}\right), k t_{s}\right)\right)^{\#} B_{d} \tilde{u}\left(k t_{s}\right)\right\|\right)
$$




$$
\begin{aligned}
& {\left[\begin{array}{c}
k \in N \\
{\left[\begin{array}{c}
y\left(k t_{S}\right) \\
\tilde{u}\left(k t_{S}\right)
\end{array}\right]=C_{d_{R}} A_{d_{R}}^{k} x_{R_{O}}+\Phi_{d_{u_{R}}}\left(k t_{S}\right) \bar{u}_{R}+\Phi_{d_{w_{R}}}\left(k t_{S}\right) \bar{w}_{R}}
\end{array}\right.} \\
& u^{-} \leq\left(B_{d}+g\left(y\left(k t_{s}\right), k t_{s}\right)\right)^{\#} B_{d} \tilde{u}\left(k t_{s}\right) \leq u^{+} \\
& y^{-} \leq y\left(k t_{s}\right) \leq y^{+} \\
& {\left[\begin{array}{c}
-\infty U_{m \times 1} \\
y_{r}^{+}
\end{array}\right] \leq \bar{u}_{R} \leq\left[\begin{array}{c}
+\infty U_{m \times 1} \\
y_{r}^{+}
\end{array}\right]} \\
& -\left[\begin{array}{c}
\bar{w} \\
0
\end{array}\right] \leq \bar{w}_{R} \leq\left[\begin{array}{c}
\bar{w} \\
0
\end{array}\right] \\
& {\left[\begin{array}{l}
y^{-} \\
u^{-}
\end{array}\right] \leq x_{R_{O}} \leq\left[\begin{array}{l}
y^{+} \\
u^{+}
\end{array}\right]}
\end{aligned}
$$

where $\|\cdot\|$ is any appropriate norm. Because of the linear structure of (52) and all of the previous assumptions made, problem (53) is convex and state-of-the-art algorithms (e.g. [20], [21]) are available for its numerical solution. If $\xi \in \mathfrak{R}_{+}$is the solution to (53), then the following bound holds.

$\left\|u\left(k t_{S}\right)\right\| \leq \xi, \forall k \in N$

From the above it is clear that if (53) is solved for all possible combinations of step, ramp and parabolic inputs between $u_{R}\left(k t_{S}\right)$ and $w_{R}\left(k t_{S}\right)$, then the maximum of these solutions will be the maximum estimated norm for the control signal that is to be expected through a response due to general reference inputs, so that the system satisfies its constraints.

\section{Control system computation and implementation}

System (26) can be controlled via $\Delta P$ in (27). With respect to the notation followed in section $5, \Delta P(t)=u(t)$ and the two reference pressures $P_{r e f_{1}}$ and $P$ ref $f_{2}$ can now be computed from (27) as $P_{r e f_{1}}(t)=\frac{P_{\max }-u(t)}{2}$ and $P_{r e f_{2}}(t)=\frac{P_{\max }+u(t)}{2}$ respectively. Since there is only one output available for measurement from (26), that is the angle of rotation, in view of (29) we have $\mathrm{C}=\left[\begin{array}{ll}1 & 0\end{array}\right]$. Hence, with respect to the numerical values of the system given in section 4, system (26) can be described by (28), (29).

For the purpose of accurate modelling the above control signal was modified as follows to incorporate the non modelled actuator dynamics in (29),

$u_{S}(t):=\sigma(u(t))$

Where $\sigma(\cdot)$ is the nonlinear function representing non modelled system dynamics (e.g. pressure dynamics as discussed in section 2, delays, saturation etc), which did not taken into account in section 5 . For particular classes of $\sigma(\cdot)$, suf- ficient conditions for the stability of the overall system can be derived using Lyapunov methods [22]. Subject to the actuator limits and after conducting few open loop experimental trials we found:

$$
\sigma(u(t)):=\left\{\begin{array}{cc}
2 \times 10^{5}, & u(t)>2 \times 10^{5} \\
L^{-1}\left\{\frac{5 e^{-0.015 s}}{s+5} u(s)\right. \\
-2 \times 10^{5}, & -2 \times 10^{5} \leq u(t) \leq 2 \times 10^{5} \\
& u(t)<-2 \times 10^{5}
\end{array}\right.
$$

to be a good approximation of the system's non modelled dynamics where $\mathrm{L}^{-1}\{\cdot\}$ is the inverse Laplace transform operator [17]. Also, there is a physical limitation on the output (angle of rotation) of the system given by

$-0.5 \leq y(t) \leq 0.5$

Based in section 5.1, for the limitations (56) and (57), we can actually prove that $A d_{R}$ in section 5.1 is asymptotically stable and furthermore that the system will never saturate according to (54). More specifically based on the numerical data of our system with a sampling time $t_{S}=0.005 \mathrm{sec}$, the design parameters in (37) and (38) were selected to give satisfactory performance as $k_{p}=0.35, k_{i n}=10$. With a numerical approximation to 4 decimal digits, (36) becomes

$$
\begin{aligned}
& u\left(k t_{s}\right)=-\frac{10^{5}\left(3.1021 y_{r}\left(k t_{s}\right)-0.8042 y\left(k t_{s}\right)\right)}{\left(0.0744 y\left(k t_{s}\right)^{2}+1\right)}- \\
& -\frac{10^{5}\left(0.0402 \sum_{i=0}^{k}\left(y_{r}\left(i t_{s}\right)-y\left(i t_{s}\right)\right)\right)}{\left(0.0744 y\left(k t_{s}\right)^{2}+1\right)}
\end{aligned}
$$

Now, in order to determine the bound (54) we solve the optimisation problem (53) using the numerical routine fmincon of the Optimisation Toolbox of MATLAB. We considered all possible combinations of reference input $u_{R}\left(k t_{S}\right)$ and $w_{R}\left(k t_{S}\right)$ according to section 5.1. The only situation at which saturation may occur is subject to ramp and parabolic inputs of $u_{R}\left(k t_{S}\right)$. This again is expected since these inputs are unbounded and the system will reach its limits unavoidably. We must emphasize, that this will not be the case if these inputs are throughout the process as discussed in section 5 .

In terms of implementation, since the controller is implemented via a digital computer, we use equation (58) and the schematic of the overall system is that of Figure 4. According to Remark 2, controller (58) do not satisfy (35) for the cases of periodic/semi-periodic input reference signals. The cause of this delay in our case is evident from (57). At this point it should be stated that the delay nonlinearity $\frac{5 e^{-0.015 s}}{s+5} u(s)$ in (57) did not taken into account in the above design, but generically this will not effect the bound (54), because of the mathematical structure of (57) and the way that (53) has been posed. Therefore, exponential stability is estimated for the system. 
The simulations of system in Figure 4 subject to control law (58) were performed in Simulink of MATLAB for a series of step reference signals to be tracked with system state and output random white noise bounded disturbances with maximum magnitude 0.001 . The above simulations are shown in Figure 5a. As far as the experiment is concern, the experimental results for the tracking of a periodic square pulse reference of period $6 \mathrm{sec}$ are shown in Figure $5 \mathrm{~b}$.
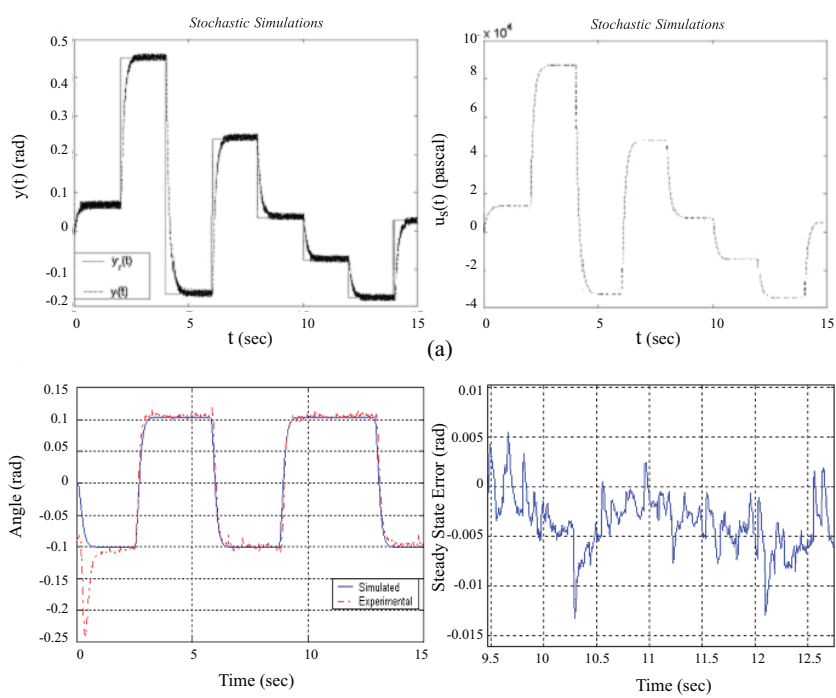

(b)

Figure 5. (a) Stochastic Simulations for the tracking of series of steps with state and measurement disturbances. (b) Experimental and respective simulation for the tracking of a square pulse signal and experimental steady state error

From figure 5, it is obvious that the simulations are in accordance with the predictions of the design method and analysis of section 5 . The method has the advantage of simplicity both in design and implementation when compared with other "modern control" designs for similar systems [13], [14] some of which produce very noisy bang-bang type control signals (see [14]) with the danger of damaging the system's actuators. It is also evident from Figure $5 b$, that there is a good match between the experimental and the theoretical step response transients with a settling time of about $0.45 \mathrm{sec}$.

\section{Conclusions / Further Work}

The modeling and control of a soft joint actuated by pneumatic muscle actuators was presented. The modeling of the joint dynamics was based on a simplified model that considers the pMAs as a nonlinear spring with elastic constant that is related to the muscle properties $\left(b, L, D_{o}\right)$. Control of the muscle pressures is achieved using inexpensive $3 / 3$ on/off solenoid valves. Comparison between the open loop response of the theoretical model and the experimental system showed good matching performance for the theoretical model. Based on the model developed a control system was designed to enable position control of the joint. The design method is characterized by its simplicity and more important by its accuracy on predicting system behavior in terms of stability and saturation avoidance. This resulted to a nonlinear controller, which shows very good performance with fast settling time. Future work include:

1. The theoretical modeling of the muscle pressure dynamics when control by means of on/off solenoid valves. This will enable the replacement of the pressure regulator identified model as described in section 3 with a theoretical model enabling the entire modeling of the system.

2. Studies about the robustness and fragility of the control system presented in this work.

3. Theoretical and experimental studies of the robustness and fragility of the control system.

4. Theoretical investigations of the design method in comparison with other modern control methods for SISO and MIMO systems and the study of possible conservatism.

5. Extension of the design in order to take into account unavoidable system saturation events [23].

\section{References}

1. R. A. Schulte, "The Characteristics of the McKibben Artificial Muscle", In the Application of External Power in Prosthetics and Orthetics, Publ. 874, Nas-RC, pp. 94-115, 1962.

2. K. Inoue, "Rubbertuator and Applications for Robots", $4^{\text {th }}$ Int. Symp. On Robotics Research, pp.57-64, Santa Cruz, CA,USA, 1987.

3. P. Chou and B. Hannaford, "Measurement and Modelling of McKibben Pneumatic Artificial Muscles"IEEE Transactions On Robotics and Automation Vol. 12, No. 1, 1996.

4. D. G. Caldwell, G. A. Medrano-Cerda, and M. J. Goodwin, "Control of Pneumatic Muscle Actuators", IEEE Control Systems Magazine, Vol. 15, No. 1, pp. 40-48, 1995

5. B. Tondu and P. Lopez, "Theory of an Artificial Pneumatic Muscle and application to the modelling of McKibben Artificial Muscle", C.R.A.S., French National Academy of science, pp. 105-114, 1996.
6. D. G. Caldwell, N. Tsagarakis, W. S. Yin and G. A. Medrano-Cerda, "Soft Actuators - Bio-mimetic Systems for a Bipedal Robot”, CLAWAR 98, pp. 279-84, Brussels, 26-28, 1998.

7. P. van der Smagt, F. Groen, K. Schulten, "Analysis and control of a rubbertuator arm", Biological Cybernetics, 75, pp. 433-440, 1996.

8. D. G. Caldwell, N. Tsagarakis, P. Artrit and G. A. Medrano-Cerda, "Biomimetic Principles in Actuator Design for a Humanoid Robot”, European $\mathrm{J}$ of Mech. and Environmental Engineering.

9. D. G. Caldwell, N. Tsagarakis, G. A. Medrano-Cerda, J. Schofield, and S. Brown, "Development of a Pneumatic Muscle Actuator driven Manipulator Rig for Nuclear Waste Retrieval Operations", IEEE Robotics and Automation Conference, Detroit, USA, May 1999.

10. N. Tsagarakis and D. G. Caldwell "Improved Modelling and Assessment of pneumatic Muscle Actuators”, ICRA 2000, San Francisco, USA May 2000. 
11. S. Davis, J. Canderle, P. Artrit, N. Tsagarakis and D. G. Caldwell, "Enhanced Dynamic Performance in Pneumatic muscle Actuators", ICRA 2002, May, Washington, D.C.

12. B. Tondu, V. Boitier and P. Lopez, "Natural compliance of robot-arms based on Mackibben artificial muscle actuators", In European robotics and Intelligent systems conference, pp. 783-797, Spain 1994.

13. P. Beomonte Zobel, T. Raparelly, G. Mattiazzo, S. Mauro, and M. Velardochia, "Force control with Fuzzy logic for Pneumatic Rubber Muscles", In Advanced Robotics Beyond 2000-The $29^{\text {th }}$ International Symposium on Robotics, Birmingham, UK, 1998.

14. P. Carbonell, Z. P. Jiang, D. Repperger, "Nonlinear Control of a Pneumatic Muscle Actuator: backstepping vs. Sliding-mode", Proc. of the IEEE International Conference on Control Applications, Méjico, D.F. (Mexico), 2001.

15. C. R. Rao, S. K. Mitra. "Generalized Inverse of Matrices and Its Applications", John Wiley, 1971.

16. T. Kailath. "Linear Systems”, Prentice Hall, 1979.
17. J. Schiff. "The Laplace Transform: Theory and Applications", Undergraduate Texts in Mathematics, Springer Verlag, 1999.

18. B. C. Kuo. "Digital Control Systems", $2^{\text {nd }}$ Ed., HBJ College \& School Division, 1995.

19. J. J. D'Azzo and C. Houpis. "Linear Control System Analysis and Design: Conventional and Modern", McGraw-Hill Series in Electrical Engineering, McGraw-Hill International, 1984.

20. R. Fletcher. "Practical Methods of Optimization", $2^{\text {nd }}$ Ed., John Wiley \& Sons, 2000

21. M. J. D. Powell. "A Fast Algorithm for Nonlinearly Constrained Optimization Calculations", Numerical Analysis, G.A.Watson ed., Lecture Notes in Mathematics, Springer Verlag, Vol. 630, 1978.

22. J. P. Lasalle. "Stability of Dynamical Systems", CBMS-NSF Regional Conference Series in Applied Mathematics 25, SIAM, 1976.

23. V. A. Tsachouridis and I. Postlethwaite. "Pseudo-linear controllers for systems with saturated actuators", IEE Proc. D. Control Theory and Applications 151, pp. 783-789, 2004 